\title{
3D FRANGI-BASED LUNG VESSEL ENHANCEMENT FILTER PENALIZING AIRWAYS
}

\author{
Daniel Jimenez-Carretero \\ Rina Dewi Rudyanto
}

\author{
Andres Santos $\quad$ Sjoerd Kerkstra \\ Maria J. Ledesma-Carbayo
}

\begin{abstract}
This paper describes a fully automatic simultaneous lung vessel and airway enhancement filter. The approach consists of a Frangi-based multiscale vessel enhancement filtering specifically designed for lung vessel and airway detection, where arteries and veins have high contrast with respect to the lung parenchyma, and airway walls are hollow tubular structures with a non negative response using the classical Frangi's filter. The features extracted from the Hessian matrix are used to detect centerlines and approximate walls of airways, decreasing the filter response in those areas by applying a penalty function to the vesselness measure. We validate the segmentation method in 20 CT scans with different pathological states within the VESSEL12 challenge framework. Results indicate that our approach obtains good results, decreasing the number of false positives in airway walls.
\end{abstract}

Index Terms - segmentation, vessel, lung, filter, CT

\section{INTRODUCTION}

Accurate quantification and visualization of lung vasculature is an important task for many clinical procedures and diagnosis, especially in cases with chronic obstructive pulmonary disease (COPD), pulmonary embolism (PE), pulmonary vascular disease (PVD), emphysema or lung cancer.

Multiple past and on-going approaches have been used for the extraction of pulmonary vessels. Appearance and geometric models [1], region growing and active contours [2], skeleton-based approaches [3] or particle filtering approaches [4] are widely used, but methods based on features extracted from the Hessian matrix $[5,6]$ are the most popular ones and they achieve quite good results in the majority of vessel scenarios. However they performed uniformly across the image and fail when other structures are locally tubular-like. Additionally, the evaluation of vascular segmentation remains a very challenging task due to complexity of the tree vessel structure and the inaccessibility to reference standard images needed to compute appropriate validation measures.

In this work we present a completely automatic method for lung vessel extraction derived from the Hessian-based filter developed by Frangi [5], modified to avoid false positives in areas that present locally tubular-like features such as the airways.

\section{METHOD}

We introduce first the principles of vessel detection based on features extracted from the Hessian matrix and the vesselness measure introduced by Frangi in section 2.1. In section 2.2 we propose a novel approach for decreasing the vesselness in airway walls. The data, experiments and evaluation results can be found in section 3 . We end with a discussion in section 4.

\subsection{Frangi Filter}

Most of Hessian-based vessel enhancement filters use eigenvalues extracted from the Hessian matrix to derive geometrical structures which can be regarded as tubular. Since vessels in the lung have different radius it is important to study these features in a multi-scale framework.

The Hessian matrix in the point $x$ at scale $\sigma, \mathcal{H}_{\sigma}(x)$, can be efficiently computed using Gaussian derivatives:

$$
\mathcal{H}_{\sigma}(I, x)=\frac{\partial^{2} I_{\sigma}}{\partial x^{2}}=I(x) * \frac{\partial^{2} \mathcal{G}_{\sigma}(x)}{\partial x^{2}}
$$

where $I$ is the image and $\mathcal{G}_{\sigma}$ is the gaussian function with standard deviation $\sigma$.

The decomposition of the local second order structure of the image extracts the eigenvalues $\left(\left|\lambda_{1}\right| \leq\left|\lambda_{2}\right| \leq\left|\lambda_{3}\right|\right)$ and principal directions $\left(\bar{u}_{1}, \bar{u}_{2}, \bar{u}_{3}\right)$. An analysis of these features allows us to differentiate between different orientation patterns (blob-like, tubular, plate-like or no preferred direction). For our purpose, an ideal tubular structure implies $\left|\lambda_{1}\right| \approx$ $0,\left|\lambda_{1}\right| \ll\left|\lambda_{2}\right|, \lambda_{2} \approx \lambda_{3}$, with $\bar{u}_{1}$ the direction of minimal curvature (along the vessel). 


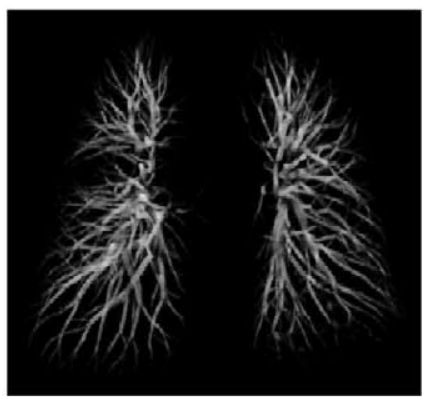

(a)

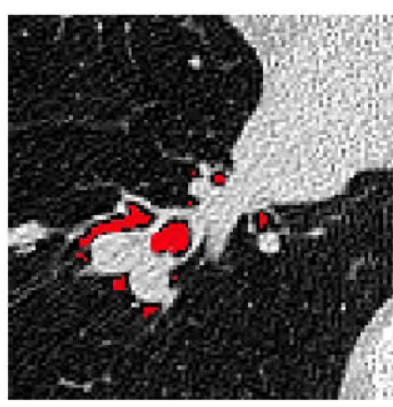

(b)
Fig. 1: (a) Rendering of vesselness extraction for lung vessels. (b) Airways enhancement (threshold $=15 \%$ for visualization).

Using this information, Frangi et al. [5] designed a vesselness function, $\mathcal{V}_{F}^{\sigma}$, to measure the similarity of one structure to an ideal tube:

$$
\mathcal{V}_{F}^{\sigma}(x)=\left\{\begin{array}{rr}
0 & \text { if } \lambda_{2}>0 \text { or } \lambda_{3}>0,{ }^{1} \\
\left(1-\exp \left(-\frac{\mathcal{R}_{\mathcal{A}}{ }^{2}}{2 \alpha^{2}}\right)\right) \exp \left(-\frac{\mathcal{R}_{\mathcal{B}}{ }^{2}}{2 \beta^{2}}\right) \\
\left(1-\exp \left(-\frac{\mathcal{S}^{2}}{2 c^{2}}\right)\right)
\end{array}\right.
$$

where $\alpha, \beta, c$ are parameters that control the sensitivity of the filter to the dissimilarity measures that distinguish between tube-like and plate-like structures $\left(\mathcal{R}_{\mathcal{A}}\right)$, blob-like $\left(\mathcal{R}_{\mathcal{B}}\right)$ and background $(\mathcal{S})$ :

$$
\begin{gathered}
\mathcal{R}_{\mathcal{A}}=\frac{\left|\lambda_{2}\right|}{\left|\lambda_{3}\right|} \\
\mathcal{R}_{\mathcal{B}}=\frac{\left|\lambda_{1}\right|}{\sqrt{\left|\lambda_{2} \lambda_{3}\right|}} \\
\mathcal{S}=\sqrt{\lambda_{1}^{2}+\lambda_{2}^{2}+\lambda_{3}^{2}}
\end{gathered}
$$

The final vesselness measure integrates the filter responses at different scales taking the maximum response:

$$
\mathcal{V}_{F}(x)=\max _{\sigma_{\min } \leq \sigma \leq \sigma_{\max }} \mathcal{V}_{F}^{\sigma}(x)
$$

Regardless of these dissimilarity measures, the filter shows a non-negative response in structures that are locally and partially tubular in the image. This is the case of airway walls, where $a$ ) they are partially tubes with the radius of wall thickness and $b$ ) sometimes they are in contact with vessels becoming indistinguishable from them.

In order to overcome these difficulties it will be necessary to link the vesselness information with the airways structure to avoid false detections in those areas. Our approach will use the features previously extracted from the classical Frangi filter to do it so.

\footnotetext{
${ }^{1}$ Condition for vessels brighter than background. For vessels darker than background the condition would be: $\lambda_{2}<0$ or $\lambda_{3}<0$
}

\subsection{Our Approach}

In order to avoid erroneous detections in airway walls, information about the bronchial tree becomes necessary. Some works noticed the importance of airway wall removal before the lung vessel segmentation step, but in most of the cases they used an independent method for airways detection and morphological operations in order to attenuate or remove airway walls $[7,8]$. Generally, those methods assumed a constant wall thickness in every bronchial generation which is not realistic. Furthermore, the additional airway segmentation increase computation times and complexity of the algorithm.

Our proposal performs vessel and airway enhancement at the same time and suggests an adaptive airway wall attenuation in order to achieve a more accurate and efficient vessel enhancement. The first step consists on bronchial tubes detection. Later, we estimate airway walls depending on the bronchial generation. Finally, a penalty function is applied in wall areas to decrease the response of the vesselness filter.

Airway enhancement. Since airways are tubular structures too, the same multi-scale filtering framework for the vessel enhancement could be used. Actually, if the scale range is appropriate (covering the possible radius of vessels and airways), a unique eigenvalue analysis of the Hessian would be necessary to extract both (Fig. 1).

Thus, we compute the vesselness function (equation 2) twice, one for the vessel extraction, $\mathcal{V}_{F v e s s e l}^{\sigma}(x)$, and one for the airways extraction, $\mathcal{V}_{\text {Fairway }}^{\sigma}(x)$. The only difference between both is the condition of being zero, changing from $\lambda_{2}>0$ or $\lambda_{3}>0$ in vessel detection (structures brighter than the background) to $\lambda_{2}<0$ or $\lambda_{3}<0$ in airways detection (structures darker than the background). To get the final measure we use equation 6 , obtaining $\mathcal{V}_{\text {Fvessel }}(x)$ and $\mathcal{V}_{\text {Fairway }}(x)$. The increment in computing time is minimum.

Airway centerline extraction. A skeletonization algorithm for 3D grayscale images based on [9] is used to extract the centerlines of bronchial tubes. It uses not only the structural information, but the density (in this case is the vesselness measure $\mathcal{V}_{\text {Fairway }}^{\sigma}(x)$ ) to obtain a more accurate estimation of centerlines.

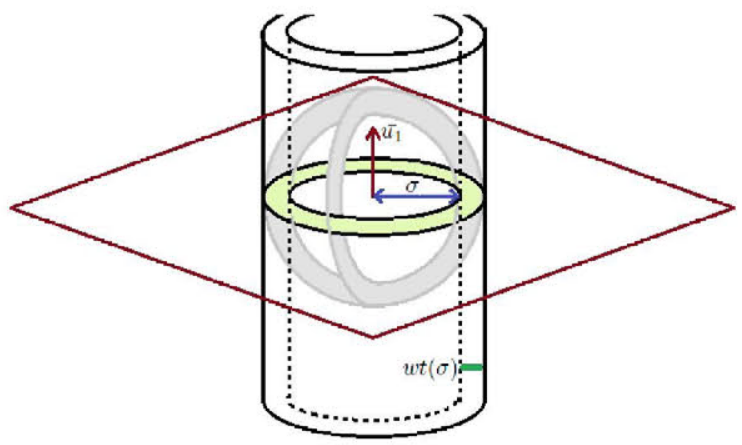

Fig. 2: Airway estimation. Wall candidates are shown in green. 
Airway wall estimation. Taking advantage of features extracted from the Hessian analysis, we can use the scale, $\sigma$, and the first eigenvector, $\overline{u_{1}}$, of maximal filter response in each point of the airway centerline. They will represent, respectively, lumen radius and directions along the vessel, so we can use them to estimate the position of the inner part of airway walls along the bronchial tubes.

In order to estimate the position of the external part of airway walls we used wall thickness values $w t(\sigma)$ measured in [10] for CT images, based on Weibel's Model [11] which depend on bronchial generation. Fig. 2 shows an scheme of airway wall estimation in one point of the bronchial centerline. Wall candidate voxels will be in the intersection between the plane with normal vector $\overline{u_{1}}$ (direction along the airway) and a hollow sphere of radius $\sigma$ and $(\sigma+w t(\sigma))$.

Penalty function. The attenuation of airway walls is performed using a penalty function over candidate voxels in the walls. Since airway detection is a pseudo-probability segmentation (not a binary one), $\mathcal{V}_{\text {Fairway }}$ measures could be interpreted as confidence of having an airway in a specific point. This information is important when the penalty function is applied to wall voxels. Thus, we subtract the airway score $\mathcal{V}_{\text {Fairway }}(y)$ obtained in one point $y$ of its centerline, to all the vessels scores $\mathcal{V}_{\text {Fvessel }}(x)$ obtained in the wall candidates for this specific point in the centerline of the bronchial tube $(x \in W$ allCandidates $(y))$ as follows:

$$
\begin{aligned}
& \mathcal{V}_{\text {Fvessel }}(x \in \text { WallCandidates }(y))= \\
& \max \left(0, \mathcal{V}_{\text {Fvessel }}(x)-\mathcal{V}_{\text {Fairway }}(y)\right)
\end{aligned}
$$

The principal idea under this penalty function is the reduction of vessel confidence depending on the airway confidence.

\section{EXPERIMENTS AND RESULTS}

\subsection{Dataset}

The dataset consists of 20 chest CT scans made available in the scope of the ISBI VESSEL12 Challenge. Details can be found on their website ${ }^{2}$.

The scans come from different scanners and protocols, using contrast media in approximately half of the CT images. They are nearly isotropic with a maximum slice spacing of $1 \mathrm{~mm}$. About half of the scans contain pathologies such as emphysema, nodules or pulmonary embolisms.

For each scan, a binary lungmask was available. They were used to reduce the scope in the vessel search. A binary erosion with a ball structuring element (radius 3 pixels) was applied and the rest of the image was fixed to the mode intensity inside the mask to reduce boundary effects in the limits of the lung.

\footnotetext{
${ }^{2} \mathrm{http} / / /$ vessel12.grand-challenge.org/
}

\subsection{Evaluation Measures}

The results have been uploaded online as a submission to VESSEL 12 challenge, and evaluated identically as the rest of the participants. A variety of axial slices were selected from each image for manual labeling. In each slice, a large number of points within the lung fields (local maxima and ramdom points) were labeled by 3 observers as: vessel, lungparenchyma, fissure, airway/airwaywall or lesion.

ROC curve analysis were used to evaluate the performance of the method. Different probability threshold values were used to compute true/false positives and true/false negatives and create points in the ROC curve. Finally, the area under the ROC curve $A z$ and specifity/sensitivity at optimal threshold were computed.

\subsection{Results}

For the experiments we use a medium range of scales ( $\sigma=$ $\{0.5,1,1.5, \ldots, 3.5\}$ ) and parameters $\alpha=\beta=0.5, c=500$. The filtered output was normalized to the range $[0,255]$.

Table 1 summarizes evaluation results on the dataset. Mean areas under the ROC curves $A z(0.978 \pm 0.013)$ show quite good performance of the algorithm in vessel detection, and values of specificity $(0.900 \pm 0.087)$ and sensitivity $(0.973 \pm 0.024)$ at optimal threshold are higher than 0.9 in almost all cases. As it is expected, scores become lower when vessels are smaller due to the size of the filter is related to the quantity of information used to evaluate the vesselness score. Moreover, contrast in small vessels is low. This makes the detection of high generation vessels a hard task.

Results for vessels/airways walls are lower than the visual examination made us expect (Figure 3). The classical Frangi filter obtains values of $A z$ between 0.738 and 0.906 depending on the scales selection, specificity values between 0.380 and 0.725 and sensitivity values between 0.889 and 0.977 . Our method outperforms these values slightly using

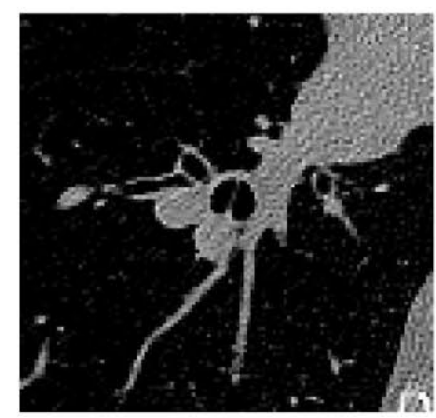

(a)

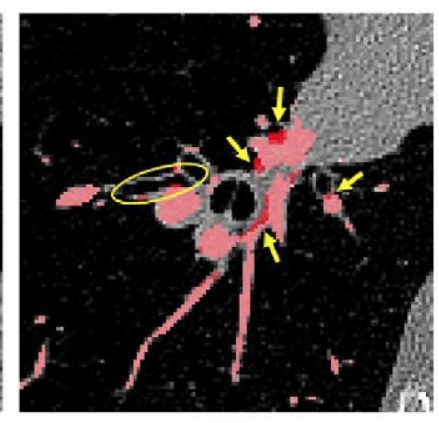

(b)
Fig. 3: (a) Example of critical area with vessels and airway walls in contact. (b) Segmentations obtained using Frangi filter (red) and our approach (white). Light pink is shown in common areas. Yellow arrows and elipse point to improvement areas. Threshold $=5 \%$ of maximum vesselness response. 
Table 1: Area under the ROC curve (Az), specificity and sensitivity at optimal threshold (from 0 to 1 ) results on the dataset using positive-points/negative-points as reference standard

\begin{tabular}{lrrr}
\hline & $A z$ & Specificity & Sensitivity \\
\hline All vessels/Non-vessels & 0.978 & 0.900 & 0.973 \\
Small vessels/Non-vessels & 0.947 & 0.887 & 0.953 \\
Medium vessels/Non-vessels & 0.985 & 0.968 & 0.953 \\
Large vessels/Non-vessels & 0.987 & 0.991 & 0.965 \\
Vessels/Airways walls & 0.918 & 0.756 & 0.973 \\
\hline
\end{tabular}

the same scales vector. Nevertheless, the evaluation framework uses a relatively low amount of positive and negative points (compared with number of voxels involved), so the validation maybe is a little limited and broader specific studies could improve these results.

A visual evaluation of segmentation results could assess strongly the contribution of this method in airway wall areas. An analysis of vessel connectivity sometimes shows incorrect connections between different vessel branches when airways wall removal is not applied. Our approach avoids many of the false positives in wall areas, correcting these mis-connections that could influence obtaining a more reliable vessel tree as well as other derived connectivity based measures

Moreover, our method could simplify estimations of airway lumen area/arterial area (LA/AA ratio), a critical marker of bronchiectasis in, for example, cystic fibrosis studies.

\section{DISCUSSION AND CONCLUSIONS}

In this paper we have presented a novel approach to enhance lung vessels simultaneously to airway in CT scans. An existing method for vessel enhancement was improved to reduce false positives in airways walls, obtaining good results in vessel detection and airway wall exclusion. Since mean $A z$ shows good results discerning vessels and airways walls, there is room for improvement in specificity and sensitivity. Additionally, a new vesselness function specifically designed for airway enhancement or the independent selection of parameters for vessel and airway segmentations could improve airway lumen detection and consequently, airway wall estimation.

In studies where a connectivity analysis is important, our method could achieve more reliable segmentations of vessels. Its use could be also critical when a separation between veins and arteries is needed or in irrigation studies assessing which vessel branches supply blood to specific lung areas.

Finally, this approach could have a direct application in bronchiectasis studies where a reliable LA/AA ratio is critical.

\section{REFERENCES}

[1] H. Shikata, E.A. Hoffman, and M. Sonka, "Automated segmentation of pulmonary vascular tree from 3D CT images," in Proc. SPIE Int. Symp. Medical Imaging, 2004, vol. 5369, pp. 107-116.

[2] L. M. Lorigo, O. D. Faugeras, W. E. L. Grimson, R. Keriven, R. Kikinis, A. Nabavi, and C.-F. Westin, "Curves: Curve evolution for vessel segmentation," Medical Image Analysis, vol. 5, pp. 195-206, 2001.

[3] T. Tozaki, Y. Kawata, N. Niki, H. Ohmatsu, and N. Moriyama, "3-D visualization of blood vessels and tumor using thin slice CT images," in Nuclear Science Symposium and Medical Imaging Conference. IEEE, 1994, vol. 3, pp. 1470-1474.

[4] R.S.J. Estepar, J.C. Ross, K. Russian, T. Schultz, G.R. Washko, and G.L. Kindlmann, "Computational vascular morphometry for the assessment of pulmonary vascular disease based on scale-space particles," in Biomedical Imaging (ISBI), 2012 9th IEEE International Symposium on. IEEE, 2012, pp. 1479-1482.

[5] A. Frangi, W. Niessen, K. Vincken, and M. Viergever, "Multiscale vessel enhancement filtering," Medical Image Computing and Computer-Assisted InterventationMICCAI98, pp. 130-137, 1998.

[6] Y. Sato, S. Nakajima, N. Shiraga, H. Atsumi, S. Yoshida, T. Koller, G. Gerig, and R. Kikinis, "Three-dimensional multi-scale line filter for segmentation and visualization of curvilinear structures in medical images," Medical Image Analysis, vol. 2, no. 2, pp. 143-168, 1998.

[7] E. van Dongen and B. van Ginneken, "Automatic segmentation of pulmonary vasculature in thoracic CT scans with local thresholding and airway wall removal," in Biomedical Imaging: From Nano to Macro, 2010 IEEE International Symposium, 2010, pp. 668-671.

[8] C. Fetita, P.Y. Brillet, and F.J. Prêteux, "Morphogeometrical approach for $3 \mathrm{~d}$ segmentation of pulmonary vascular tree in multi-slice CT," in SPIE Medical Imaging, 2009, vol. 7259, pp. 72594F-72594F.

[9] N. Gagvani and D. Silver, "Parameter-controlled volume thinning," Graphical Models and Image Processing, vol. 61, no. 3, pp. 149-164, 1999.

[10] M. Montaudon, P. Desbarats, P. Berger, G. De Dietrich, R. Marthan, and F. Laurent, "Assessment of bronchial wall thickness and lumen diameter in human adults using multi-detector computed tomography: comparison with theoretical models," Journal of Anatomy, vol. 211, no. 5, pp. 579-588, 2007.

[11] E.R. Weibel, B. Sapoval, and M. Filoche, "Design of peripheral airways for efficient gas exchange," Respiratory Physiology \& Neurobiology, vol. 148, no. 1, pp. 3-21, 2005. 